\title{
Solving a bicriterion scheduling problem
}

\author{
Luc N. VAN WASSENHOVE * and \\ Ludo F. GELDERS \\ Katholieke Universiteit Leuven, Industrieel Beleid, \\ B-3030 Heverlee, Belgium
}

Received October 1978

Revised December 1978

Consider $n$ jobs to be sequenced ofi a single machine. The objective functions to be minimized are the holding cost and the maximum tardiness. We first characterize the set of efficient points and then proceed to give a pseudo-polynomial algorithm to enumerate all these efficient points. Computational results illustrate the usefulness of the procedure.

\section{Introduction}

A major conclusion of Panwalkar's repurt [6] on industrial scheduling was that managers do schedule according to multiple objectives. Nevertheless, scheduling research continued to deal predominantly with a single objective. It is clear however that a socalled optimum with respect to one objective, could perform extremely bad with respect to other criteria.

Therefore, a 'non-optimal' solution with satisfactory performance on other measures might be considered as a better alternative by the decision maker. This point was partly recognized by some researchers who studied scheduling problems with secondary criteria (see $[2,7,8]$ among others). These studies identify the best sequence for the secondary measure frcm among the set of alternate optima with respect to the primary measure.

In this paper we address the bicriterion scheduling problem. The two criteria we consicer are the minimization of flowtime and the minimization of maximum tardiness. Though we realize that most (single objective) scheduling problems are combinatorially hard, we hope that this work will provide an incentive for an increased research effort into the field of multicriteria scheduling problems.

Section 2 presents the problem formulation. In

* Sponsored by the Doctoral College in Management (C.I.M.), Eendrachtstraat 53, 1050 Brussels, Belgium.

(c) North-Holland Publishing Company

European Journal of Operational Research 4 (1980) 42-48.
Section 3 efficient solutions are characterized. Section 4 presents an algorithm to identify all efficient points. In Section 5 we analyze the complexity of the algorithm. Section 6 yields computational results. Finally, in Section 7 some conclusions and suggestions are given.

\section{A bicriterion scheduling problem}

Consider the problem of sequencing $n$ jobs on a single processor. All jobs are simultaneously available and are charactesized by their processing time $p_{i}$ and due date $d_{i}$. The decision maker has two objectives in mind. The first one is to minimize flowtime (a measure for average in-process-inventory). The second one deals with service to the customers for which maximum tardiness is chosen as an appropriate measure.

Let

i1: the set of permutation schedules,

$\pi$ : a permutation schedule,

$C_{i}$ : the completion time of job $i$ (given a perrnutation schedule),

$T(\pi)$ : the maximum tardiness of schedule $\pi$,

$H(x)$ : the total flowtime of schedule $\pi$.

Then, the problem (P) can be written as:

$\min _{\pi \in \Pi} \sum_{i=1}^{n} C_{i}=\min _{\pi \in \Pi} H(\pi)$,

(P)

$\min _{\pi \in \Pi} \max _{i=1, \ldots, n}\left\{\max \left(0, C_{i}-d_{i}\right)\right\}=\min _{\pi \in \Pi} T(\pi)$.

The decision maker knows that objective (1) is reached in at most $O(n \log n)$ steps by ordering the jobs according to nondecreasing processing times (SPT-rule). He is also aware of the fact that (2) is obtained in at most $\mathrm{O}(n \log n)$ steps by ordering the jobs according to nondecreasing due dates (EDDrule). None of these rules is a priori appealing to him because he knows from experience that they usually perform rather poorly when both objectives are to be considered. He is interested in a sequence that 'coes well' on both objectives (if such a sequence exists). In order: to define such a sequence more precisely, we turn to the concept of efficiency. 
A sequence $\pi^{*} \in \Pi$ is efficient in problem $(\mathrm{P})$ if there exists no $\pi \in \Pi$ such that

$H(\pi) \leqslant H\left(\pi^{*}\right)$ and $T(\pi) \leqslant T\left(\pi^{*}\right)$

where at least one relation holds with strict inequality.

Sinilarly, we say that a sequence $\pi_{1}$ dominates a sequence $\pi_{2}$ when

$H\left(\pi_{1}\right) \leqslant H\left(\pi_{2}\right)$ and $T\left(\pi_{1}\right) \leqslant T\left(\pi_{2}\right)$

where at least one relation is a strict inequality.

Clearly, the decision maker will choose an efficient sequence. Therefore, the researcher's problem is to characterize the set of efficient sequences and to help the decision maker in his search through this set in order to decide upon a final sequence to be implemented.

\section{Characterizing efficient solutions}

Proposition 1. There exists an efficient sequence in the bicriterion problem $(\mathrm{P})$ that satisfies the SPT-rule.

Proof. (a) Suppuse first that all processing times are different. The unique SPT-sequence (SPT ${ }^{*}$ ) gives the absolute minimum of $H$. Hence, there is no sequerice $\pi \neq$ SPT $^{*}$ such that

$H(\pi) \leqslant H\left(\mathrm{SPT}^{*}\right)$ and $T(\pi) \leqslant T\left(\mathrm{SPT}^{*}\right)$

with at least one strict inequality since (3) cannot hold.

(b) If more than one SPT-sequence exists (jobs with equal processing times), let SFT ${ }^{*}$ be a sequence satisfying the SPT-rule and surh that jobs with equal processing times are in EDD-sequence. Note that if several jobs have identical processing times and identical due dates, SPT $^{*}$ is not unique. We prove that every SPT ${ }^{*}$-sequence is efficient - sequences that do not satisfy the SPT-rule cannot dominate an SPT* sequence (3) - if $\pi$ is an SPT-sequence but not an $\mathrm{SPT}^{*}$-sequence, it cannot dominate SPT ${ }^{*}$ since

$H\left(\mathrm{SPT}^{*}\right)=H(\pi)$ and $T\left(\mathrm{SPT}^{*}\right) \leqslant T(\pi)$

by virtue of the EDD-rule.

Henc $^{\circ}$, all SPT ${ }^{*}$-sequences are efficient.

Fig. 1 shows the effect of Proposition 1 in the ob. jective function space.

Note that an analogous proposition for the EDD. rule does not hold in general as shown by the follow. ing example where, as before, $p_{i}$ denotes the pro-

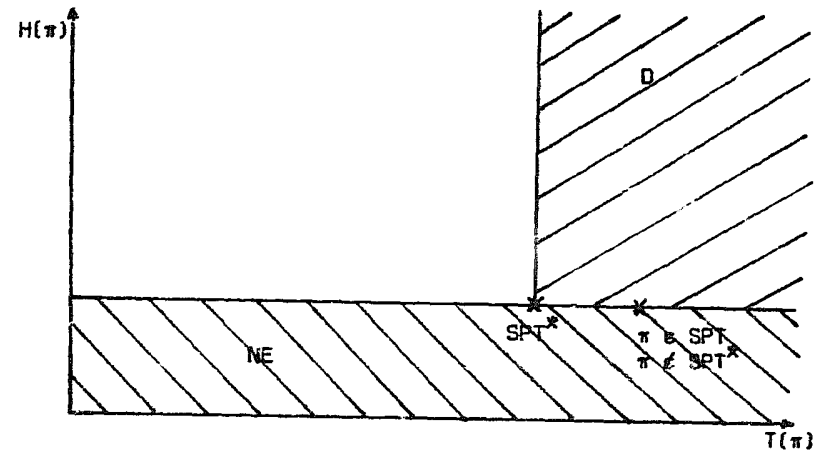

Fig. 1. Proposition 1. (D = doninated, $N E=$ non-existirg).

cessing time and $d_{i}$ denotes the due date of job $i$ :

\begin{tabular}{lll}
\hline$i$ & $p_{i}$ & $d_{i}$ \\
\hline 1 & 2 & 3 \\
2 & 3 & 4 \\
3 & 1 & 5 \\
4 & 2 & 6 \\
\hline
\end{tabular}

$\mathrm{SPT}^{*} \rightarrow$ sequence $3.14-2, H\left(\mathrm{SPT}^{*}\right)=17, T\left(\mathrm{SPT}^{*}\right)=4$ $\mathrm{EDD} \rightarrow$ sequence $1-2.34, H(E D D)=21, T(E D D)=2$ SPT $^{*}$ is efficient by Proposition 1.

EDD is not efficient since it is dominated by sequence 3.1.2.4 with $H=18$ and $T=2$.

Proposition 2. Let $\Delta$ be an integer such thet $T(E D D)$ $\leqslant \Delta$ and put $\cdot D_{i}=d_{i}+\Delta$, for all $i$.

Define problem $(\mathrm{P} 1)$ as:

$\min _{\pi \in \Pi} \sum_{i=1}^{n} C_{i}$

(P1)

$C_{i} \leqslant D_{i}, \quad$ all $i$.

Then, at least one optimal solution to $(\mathrm{P} 1)$ is efficient in $(\mathbb{P})$, where $(\mathbb{P})$, as before, denotes the bicriterion problem of minimizing holding cosi and risurimum tardiness.

Proof. (PI) is solved by Smith's backward sched. uling rule [7], namely
(i) Set $R=\sum_{i=1}^{n} p_{i}, N=\{1, \ldots, n\}, k=n$, 
(ii) Find a job $j^{*} / p_{j^{*}} \geqslant p_{j}, D_{j^{*}}, D_{j} \geqslant R, j, j^{*} \in N$ (break ties arbitrarily).

Assign job $j^{*}$ to position $k$.

(iii) Set $R=R-p_{j^{*}}, N=N-\left\{j^{*}\right\}, k=k-1$. If $k=0$, stop. Else go to (ii).

This means that at each decision point the set of scheduleable jobs is inspected (i.e. these jobs that can be scheduled without violating their due dates) and the largest job is selected to be scheduled next. The procedure uses backward loading, i.e. it always decides upon a job to be placed in the last position.

Modify Smith's procedure as follows: break ties in step (ii) by selecting the job with the largest due date. That is, if there is more than one 'largest' scheduleable job, we want the one with the largest due date to be performed last. We prove that any solution $\pi^{*}$ satisfying the modified Smith procedure is efficient in problem (P). Let $\pi_{1}$ be any feasible solution to (P1) not satisfying the modified Smith rule; $\pi_{2}$ be any infeasible solution to (P1). Then, if $\pi_{1}$ does not satisfy Smith's rule, we have

$H\left(\pi^{*}\right)<H\left(\pi_{1}\right)$.

If $\pi_{1}$ satisfies Smith's rule but not the modified Smith rule, we have:

$H\left(\pi^{*}\right)=H\left(\pi_{1}\right)$ and $T\left(\pi^{*}\right) \leqslant T\left(\pi_{1}\right)$

by virtue of the embedded EDD-rule. Hence $\pi_{1}$ can never dominate $\pi^{*}$.

We also know that $T\left(\pi^{*}\right)<T\left(\pi_{2}\right)$ since $\pi_{2}$ is infeasible in (P1). Therefore $\pi_{2}$ can never dominate $\pi^{*}$.

Fig. 2 shows the effect of Proposition 2 in the objective function space.

Remarks. (1) Proposition 1 is a special case of Proposition 2 for $\Delta$ large enough, e.g. putting $\Delta=\sum_{i=1}^{n} p_{i}$ yields $D_{i}=d_{i}+\sum_{i=1}^{n} p_{i} \geqslant \sum_{i=1}^{n} p_{i}$ for all $i$ (assuming

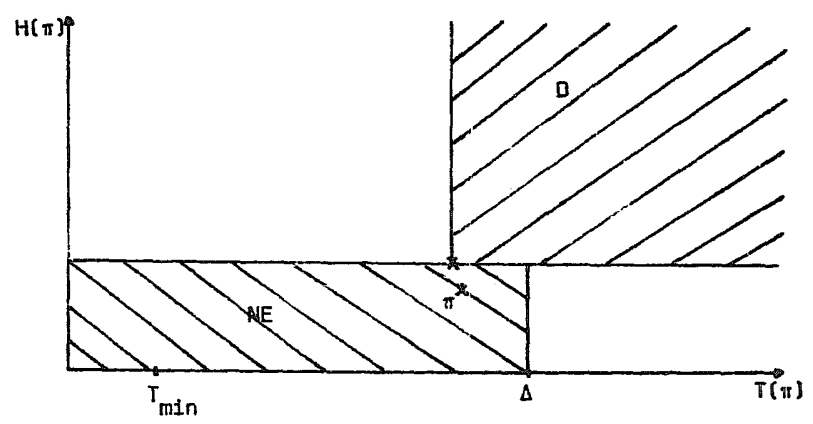

Fig. 2. Proposition 2. $(D=$ dominated, $N E=$ non-existing). $d_{i} \geqslant 0$, all $i$ ). Hence, in step (ii) of the modified Smith procedure, all jobs can be selected (the due date restriction becomes irrelevant). As a result, the procedure will generate an SPT ${ }^{*}$-sequence.

(2) It is possible for a sequence satisfying Smith's rule but not the modified Smith rule to be efficient. Such a sequence would have holding costs and maximum tardiness identical to a sequence satisfying the modified Smith rule. In order to circumvent these 'degeneracies' we will further consider sequences with the same objective function values to be identical (i.e. the decision maker is indifferent to such sequences).

(3) Proposition 2 suggests an algorithm for finding all efficient points in problem (P) (i.e. all efficient combinations of $H$ and $T$ ) and an efficient sequence for each point by iteratively changing the value of $\Delta$. Such an algorithm is given below.

\section{An algorithm}

Step 0. Put $\Delta=\sum_{i=1}^{n} p_{i}$.

Step 1. Let $D_{i}=d_{i}+\Delta$ for all $i$.

Step 2. Solve (P1) using the modified Smith rule. If a solution ( $\left.\pi^{*}\right)$ exists, then it is efficient (Proposition 2). Else, go to Step 4.

Step 3. Compute $T\left(\pi^{*}\right)=\max _{i=1}, \ldots, n\{\max (0$, $\left.\left.C_{i}-d_{i}\right)\right\}$. Put $\Delta=T\left(\pi^{*}\right)-1$. Go to Step 1 .

Step 4. Stop.

It is easy to see that the algorithm will generate all efficient points. The procedure is illustrated on an example from Conway, Maxwell and Miller [1, p.32].

\begin{tabular}{lll}
\hline $\boldsymbol{i}$ & $\boldsymbol{p}_{\boldsymbol{i}}$ & $\boldsymbol{d}_{\boldsymbol{i}}$ \\
\hline 1 & 2 & 1 \\
2 & 4 & 2 \\
3 & 3 & 4 \\
4 & 1 & 6 \\
\hline
\end{tabular}

Step 0: $\Delta=\Sigma p_{i}=10$;

Step 1: $D=\left\{\boldsymbol{D}_{i}\right\}=(11,12,14,16)$;

Step 2: $\pi^{*}=4$-1-3-2 (SPT-sequence), $H\left(\pi^{*}\right)=20$;

Step 3: $T\left(\pi^{*}\right)=8, \Delta=8-1=7$;

Step 1: $D=(8,9,11,13)$;

Step 2: $\pi^{*}=4-1-2-3, H\left(\pi^{*}\right)=21$;

Step 3: $T\left(\pi^{*}\right)=6, \Delta=6-1=5$;

Step 1: $D=(6,7,9,11)$; 
Table 1

Efficient sequences for the bicriterion problem of minimizing $H$ and $T$

\begin{tabular}{llll}
\hline SEQUENCE & $H$ & $T$ & $\Sigma T$ \\
\hline SPT & 20 & 8 & 12 \\
$4-1-2-3$ & 21 & 6 & 13 \\
EDD & 27 & 5 & 14 \\
\hline
\end{tabular}

Step 2: $\pi^{*}=1-2-3-4$ (EDD-sequence), $H\left(\pi^{*}\right)=27$;

Step 3: $T\left(\pi^{*}\right)=5, \Delta=5-1=4$;

Step 1: $D=(5,6,8,10)$;

Step 2: Infeasible;

Step 4: Stop.

Table 1 shows the results for this example. The table includes the sum of iardiness $(\Sigma T)$ for illustrative purposes. It follows from Table 1 that the decision maker would probably choose sequence 4-1-2-3.

Fig. 3 shows all $(n !=24)$ permutation schedules in the objective function space.

\section{Complexity}

We now investigate the time complexity of the above algorithm. Both Steps 1 and 3 are of $O(n)$. Smith's procedure (Step 2) can be carried out in $O(n \log n)$ when the jobs are preordered in SPT-

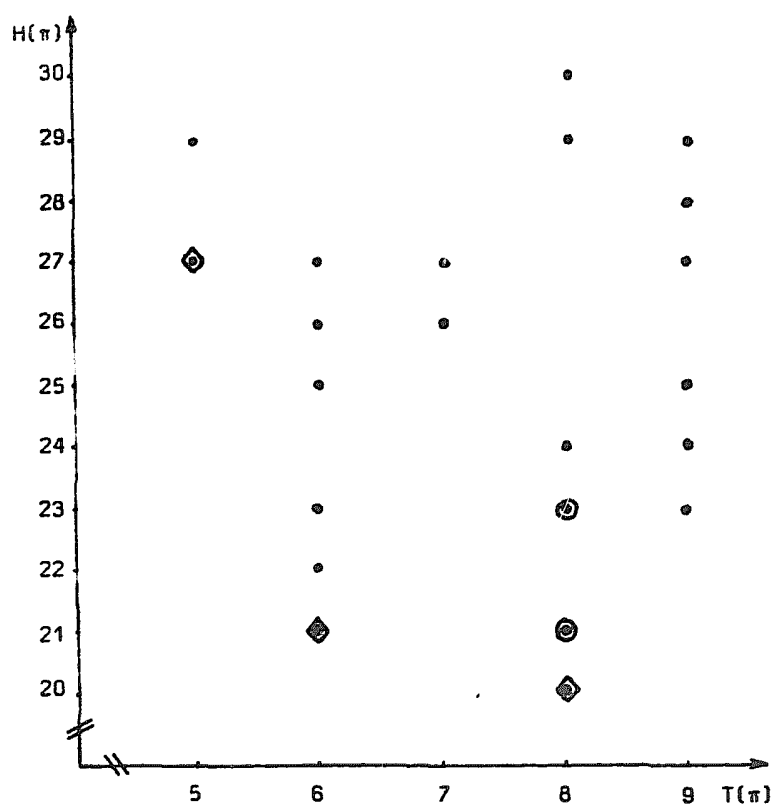

Fig. 3. Complete enumeration for the example problem $(\odot=2$ coinciding points, $\diamond=$ efficient points). sequence. We further assume that

$0 \leqslant \min _{i} d_{i} \leqslant \max _{i} d_{i} \leqslant \sum_{i} p_{i}$

Now

$T(\mathrm{EDD}) \geqslant \sum_{i} p_{i}-\max _{i} d_{i} \geqslant 0$,

$T\left(\mathrm{SPT}^{*}\right) \leqslant \sum_{i} p_{i}-\min d_{i} \leqslant \sum_{i} p_{i}$

or

$$
\begin{aligned}
T\left(\mathrm{SPT}^{*}\right)-T(\mathrm{EDD}) & \leqslant \max _{i} d_{i}-\min _{i} d_{i} \\
& \leqslant r \quad \text { (range of due dates). }
\end{aligned}
$$

Also

$T\left(\mathrm{SPT}^{*}\right)-T(\mathrm{EDD}) \leqslant \sum_{i} p_{i}$

The number of times that Steps 1 to 3 have to be executed is at most $T\left(\mathrm{SPT}^{*}\right)-T(\mathrm{EDD})+2(8-5+$ $2=5$ in the example problem).

From (5) it follows that $T\left(\mathrm{SPT}^{*}\right)-T(\mathrm{EDD})=$ $\mathrm{O}\left(\Sigma_{i} p_{i}\right)$

Therefore, the overall time-complexity of the algorithm is:

$\mathrm{O}\left(n \log n \cdot \sum_{i=1}^{n} p_{i}\right)$

or, taking $\bar{p}$ as the average processing time:

$\mathrm{O}\left(n^{2} \bar{p} \log n\right)$.

Strictly speaking, the complexity is not polynomial since it depends on $\bar{p}$ (pseudo-polynomial). However, for reasonable values of $\bar{p}$, it can be considered as polynomial. More precisely: if $p_{i} \leqslant p^{*}$ (all $\left.i\right)$, then the complexity is $O\left(n^{2} p^{*} \log n\right)$.

From (4) it follows that the time complexity can also be expressed as $O(r n \log n)$ where $r$ is the range of due dates.

\section{Computational results}

The algorithm of Section 4 was coded in FORTRAN IV and tested on IBM 370/158. Data were generated at random from uniform distributions with $1 \leqslant p_{i} \leqslant 10$ and $0 \leqslant d_{i} \leqslant \Sigma p_{i}$.

A typical example is given in Table 2. The results are summarized in Table 3. 
Table 2

Data

\begin{tabular}{|c|c|c|c|c|c|c|c|c|c|c|}
\hline & 1 & 2 & 3 & 4 & 5 & 6 & 7 & 8 & 9 & 10 \\
\hline $\begin{array}{c}p_{i} \\
d_{i}\end{array}$ & $\begin{array}{r}9 \\
32\end{array}$ & $\begin{array}{r}9 \\
49\end{array}$ & $\begin{array}{l}6 \\
7\end{array}$ & $\begin{array}{r}7 \\
25\end{array}$ & $\begin{array}{r}2 \\
55\end{array}$ & $\begin{array}{l}4 \\
9\end{array}$ & $\begin{array}{r}7 \\
54\end{array}$ & $\begin{array}{r}2 \\
40\end{array}$ & $\begin{array}{r}7 \\
52\end{array}$ & $8^{8^{a}}$ \\
\hline
\end{tabular}

${ }^{2} \Sigma p_{i}=61$.

Results for 10,30 and 50-job problems are given in Table 4. Because of page limitations, only five examples are given for each problem size. Extensive results can be obtained from the authors.

Table 4 should be read as follows:

NEP = the actual number of efficient points for the problem at hand. Remember that the procedure finds all these points.

MNEP = maximum possible number of efficient points for the problem at hand. Since an efficient point could exist for each value of the maximum tardiness between $T\left(\mathrm{SPT}^{*}\right)$ and $T$ (EDD), this maximum possible number equals $T\left(\mathrm{SPT}^{*}\right)-T(\mathrm{EDD})+1$.

$H(E D D), T(E D D))=$ the criterion vector for the EDD-sequence

$\left(H\left(\mathrm{SPT}^{*}\right), T\left(\mathrm{SP}^{*}\right)\right)=$ the criterion vector for the $\mathrm{SPT}^{*}$-sequence

$\left(H\left(\pi^{*}\right), T\left(\pi^{*}\right)\right)=$ the criterion vector for the selected efficient point. In order to choose this efficient point from among the set of efficient points we put on our decision maker's hat and simply picked the point that looked most attractive to us in ter ns of the trade-off between the two objective functiorss. That is, the procedure generates all efficient points and we choose one by inspection. For the example in Table 3 we chose $\left(H\left(\pi^{*}\right), T\left(\pi^{*}\right)\right)=(274,7)$ corresponding to efficient point number 4 . Obviously, this choice depends upon the decision maker's preferences. Nevertheless, the point $(\mathbf{2 7 4 , 7 )}$ will look

Table 3

\begin{tabular}{lrrl}
\hline & $H$ & $T$ & SEQUENCE \\
\hline EDD & 341 & 6 & $3-6-4-1-8-2-10-9-7-5$ \\
EFFICIENT POINTS & & & \\
$1\left(\right.$ SPT $\left.^{*}\right)$ & 268 & 20 & $8-5-6-3-4-9-7-10-1-2$ \\
2 & 269 & 12 & $8-5-6-3-4-9-7-1-10-2$ \\
3 & 272 & 10 & $8-5-6-3-4-9-1-7-2-10$ \\
4 & 274 & 7 & $8-5-6-3-4-9-1-10-2-7$ \\
5 & 315 & 6 & $8-6-3-4-9-1-10-2-7-5$ \\
\hline
\end{tabular}

attractive to most decision makers concerned with optimizing both objectives since it almost minimizes maximum tardiness (7 vs. 6 ) while it only slightly increases the holding cost (274 vs. 268).

There are several reasons why we decided not to include a systematic procedure for choosing an effcient point from the generated set of efficient points. First of all, such procedures are available in the literature (ree e.g. $[5,9]$ ) and the selection of an appropriate one depends upon the decision maker and the available computer configuration (e.g. interactive or not). Secondly, most existing procedures establish some overall utility function for the decision maker at the outset. This is a cumbersome and delicate task. Usually the decision maker is asked to express his indifference towards cercain trade-offs between the objectives, the resulting answers should then be carefully checked for consistency. Such an elaborate procedure might certainly be warranted for important strategic decisions (e.g. nvestment decisions) where monetary trade-offs can be evaluated fairly easily by the decision maker. Scheduling decisions, however, are mostly routine decisions with a high frequency of occurrence, each individual decision being only moderately irnportant. Uttermost important to the shop floor manager is a fast and reliable procedure that allows him to take a fairly good decision (fairly good with respect to strict mathematical optimality, the decision might be very good considering time pressure, imperfect information and aspects not included in the model). Moreover, it is doubtful whether the shop floor manager can (or wants to) answer questions like: "Are you indifferent to a flowtime of 164 and s maximurn tardiness of 24 on one hand and a flowtime of 212 and maximum tardiness of 12 on the other." We believe that for the type of scheduling problems we consider the best thing to do is to present the set of efficient points to the shop floor manager and to let him make his own choice. From Table 4 it follows that the set of efficient points is usually fairly restricted. Moreover, the decision maker can use 
Table 4

Computational results

\begin{tabular}{|c|c|c|c|c|c|c|c|}
\hline $\begin{array}{l}\text { Problem } \\
\text { Size }\end{array}$ & $\begin{array}{l}\text { Problem } \\
\text { Number }\end{array}$ & NEP & MNEP & $(H(E D D), T(E D D)$ & $\left(H\left(\mathrm{SPT}^{*}\right), T\left(\mathrm{SPT}^{*}\right)\right)$ & $\left(H\left(\pi^{*}\right), T\left(\pi^{*}\right)\right)$ & $\begin{array}{l}\text { CPU-Time } \\
\text { (ms) }\end{array}$ \\
\hline \multirow{5}{*}{10} & 1 & 3 & 6 & $(278,6)$ & $(212,11)$ & $(232,8)$ & 6 \\
\hline & 2 & 4 & 10 & $(242,2)$ & $(219,11)$ & $(223,2)$ & 7 \\
\hline & 3 & 5 & 15 & $(341,6)$ & $(268,20)$ & $(274,7)$ & 10 \\
\hline & 4 & 10 & 27 & $(294,13)$ & $(216,39)$ & $(243,16)$ & 17. \\
\hline & 5 & 14 & 32 & $(332,10)$ & $(246,61)$ & $(252,20)$ & 25 \\
\hline \multirow{5}{*}{30} & 1 & 12 & 47 & $(2227,55)$ & $(1761,101)$ & $(1765,77)$ & 73 \\
\hline & 2 & 17 & 41 & $(2804,88)$ & $(1975,128)$ & $(2014,88)$ & 103 \\
\hline & 3 & 16 & 42 & $(2578,70)$ & $(1903,111)$ & $(1906,94)$ & 95 \\
\hline & 4 & 25 & 80 & $(2408,61)$ & $(1733,140)$ & $(1750,83)$ & 147 \\
\hline & 5 & 21 & 34 & $(2619,74)$ & $(1926,106)$ & $(1968,90)$ & 123 \\
\hline \multirow{5}{*}{50} & 1 & 9 & 17 & $(6300,145)$ & $(4246,161)$ & $(4264,147)$ & 131 \\
\hline & 2 & 21 & 71 & $(6672,161)$ & $(4648,231)$ & $(4702,162)$ & 288 \\
\hline & 3 & 29 & 62 & $(6547,169)$ & $(5218,230)$ & $(5302,173)$ & 394 \\
\hline & 4 & 17 & 58 & $(6007,145)$ & $(4362,202)$ & $(4396,145)$ & 243 \\
\hline & 5 & 12 & 62 & $(6619,166)$ & $(5047,227)$ & $(5129,166)$ & 168 \\
\hline
\end{tabular}

some simple rules to restrict this set even more. After some efficient points have been generated, the decision maker could use this information to place upper and lower bounds on the objective function values. The procedure can easily be adapted to generate only the efficient points satisfying these bounds. Similarly, the decision maker could consider a third objective and only be interested in efficient points with a certain minimum score on the third cbjective. Again, the procedure is easily adapted to presert only these points to the decision maker.

The attractiveness and practicality of our procedure relies upon its simplicity. Decisions can be made 'on the spot' by the use of a somewhat sophisticated pocket calculator or a small decentralized computing machine. We realize that the procedure lacks elegance because it does not terminate with 'the' optimal choice. On the other hand, we consider the shop floor manager to be capable to select the most appropriate point from a restricted set of alternatives.

\section{Discussion}

We proved that the SPT-rule can easily be adapted for obtaining an efficient point. A counterexample showed that the EDD-rule does not necessarily yield an efficièiitî point. Both rules require at most $O(n \log n)$ steps and both rules minimize one objec- tive while completely ignoring the other. If we are willing to spend a little more computational effort $\left(\mathrm{O}\left(n^{2} p^{*} \log n\right)\right)$, we can find all efficient points. This allows for a justified choice of a sequence that takes both objective functions into account and that is not dominated by any other sequence. In particular, a slight increase in one objective function (e.g. holding cost) often results in a large decrease in the other (maximum tardiness). This important point is missed by all previous scheduling researc 4 .

From our computational results we conclude that:

(a) CPU-requirements are negligible.

(b) The number of efficient points is usually small. Therefore, the decision maker should be able to make his choice without the aid of an interactive method to guide his search.

(c) Very attractive efficient points are found for all the test problems. In most cases we discovered efricient points that were surprisingly close to the optimum value for both objectives whereas the EDD. rule and the SPT-rule performed badly on the holding cost and the maximum tardiness objective respectively.

We point out that it is probably possible to find algorithms for locating all efficient points that would work faster on the average than the one presented above. However, we feel that the (worst case) time complexity of cur algorithm is minimal. The reason is that there could be an efficient point for each 
vrdue of $T$ between $T(E D D)$ and $T\left(\mathrm{SPT}^{*}\right)$ and finding in efficient point will at least require some ordering of jobs i.e. $O(n \log n)$.

Note also that an optimal solution to a scheduling problem with a secondary criterion satisfies the definition of efficiency. These scheduling problems therefore deal with locating a single efficient point and could be viewed as a first step towards solving multicriteria scheduling problems.

This paper has dealt with a simple bicriterion scheduling problem. Our primary purpose was to exhibit the importance of multiple objective routines in solving 'the scheduling problem' as it occurs in a real-life environment and to provide an incentive for an increased research effort in this area. We are convinced that lots of multicriteria scheduling problems can be solved with existing techniques and that interesting results are to be expected from new developments in this field. As an example, consider the problem of minimizing weighted tardiness and weighted flowtime on a single processor when all processing times are equal. This problem can be formulated as a bicriterion linear program (or a bicriterion assignment problem) for which numerous techniques have already been developed $[3,4,9]$. Existing interactive codes can be used to help the decision maker in his choice of an efficient solution. The point of the matter is that a whole body of knowledge on multiple criteria optimization is available and that it should be applied on scheduling problems since scheduling prob. lems almost always involve multiple conflicting criteria.

\section{Keferences}

[1] R.W. Conway, W.L. Maxwell and L.W. Miller, Theory of Scheduling (Addison-Wesley, Reading, MA, 1967).

[2] H. Emmons, A note on a scheduling problem with dual criteria, Naval Res. Logist. Quart. 22 (1975) 615-616.

[3] J.P. Evans and R.E. Steuer, Generating efficient extreme points in linear multiple otjective programming: two algorithms and computing experience, in: J.L. Cochrane and M. Zeleny, Eds., Multiple Criteria Decision Making (University of S. Carolina Fress, Columbia, SC, 1973).

[4] A.M. Geoffrion, Proper efficiency and the theory of vector maximization, J. Math. Anal. Appl. 22 (1968) 618-630.

[5] R.L. Keeney and H. Raiffa, Decisions with Multiple Objectives: Preference and Value Trade-Offs (J. Wiley, New York, 1976).

[6] S.S. Panwalkar, R.A. Dudek and M.L. Smith, Sequencing research and the industrial scheduling problem in: S.E. Elmaghraby, Ed., Symposium on the Theory of Scheduling and its Applications (Springer, New York, 1973).

[7] W.E. Smith, Various optimizers for single-stage production, Naval Res. Logist Quart. 3 (1956) 59-66.

[8] L. Var Wassenhove, Minimizing weighted flowtime subject to deadlines, Naval Res. Logist. Quart. To appear.

[9] M. Zeleny, Linear Multiobjective Programming, Lecture Notes in Econ. and Math. Systems 95 (Springer, Berlin, 1974). 